\title{
Rapid Ultrastructural Changes in the PSD and Surrounding Membrane after Induction of Structural LTP in Single Dendritic Spines
}

\author{
Ye Sun, ${ }^{1,3,4}$ Michael Smirnov, ${ }^{1}{ }^{\circledR}$ Naomi Kamasawa, ${ }^{2,4}$ and ${ }^{\circledR}$ Ryohei Yasuda ${ }^{1,3,4}$ \\ ${ }^{1}$ Neuronal Signal Transduction Group, Max Planck Florida Institute for Neuroscience, Jupiter, Florida 33458, ${ }^{2}$ Electron Microscopy Core Facility, \\ Max Planck Florida Institute for Neuroscience, Jupiter, Florida 33458, ${ }^{3}$ Integrative Biology and Neuroscience Graduate Program, Florida Atlantic \\ University, Jupiter, Florida 33458, and ${ }^{4}$ International Max Planck Research School for Brain and Behavior, Max Planck Florida Institute for \\ Neuroscience, Jupiter, Florida 33458
}

The structural plasticity of dendritic spines is considered to be an important basis of synaptic plasticity, learning, and memory. Here, we induced input-specific structural LTP (sLTP) in single dendritic spines in organotypic hippocampal slices from mice of either sex and performed ultrastructural analyses of the spines using efficient correlative light and electron microscopy. We observed reorganization of the PSD nanostructure, such as perforation and segmentation, at 2-3, 20, and $120 \mathrm{~min}$ after sLTP induction. In addition, PSD and nonsynaptic axon-spine interface (nsASI) membrane expanded unevenly during sLTP. Specifically, the PSD area showed a transient increase at 2-3 min after sLTP induction. The PSD growth was to a degree less than spine volume growth at 2-3 min and $20 \mathrm{~min}$ after sLTP induction but became similar at $120 \mathrm{~min}$. On the other hand, the nsASI area showed a profound and lasting expansion, to a degree similar to spine volume growth throughout the process. These rapid ultrastructural changes in PSD and surrounding membrane may contribute to rapid electrophysiological plasticity during sLTP.

Key words: correlative light and electron microscopy; glutamate uncaging; LTP; structural LTP; synaptic plasticity

\section{Significance Statement}

To understand the ultrastructural changes during synaptic plasticity, it is desired to efficiently image single dendritic spines that underwent structural plasticity in electron microscopy. We induced structural long-term potentiation (sLTP) in single dendritic spines by two-photon glutamate uncaging. We then identified the same spines at different phases of sLTP and performed ultrastructural analysis by using an efficient correlative light and electron microscopy method. We found that postsynaptic density undergoes dramatic modification in its structural complexity immediately after sLTP induction. Meanwhile, the nonsynaptic axon-spine interface area shows a rapid and sustained increase throughout sLTP. Our results indicate that the uneven modification of synaptic and nonsynaptic postsynaptic membrane might contribute to rapid electrophysiological plasticity during sLTP.

\section{Introduction}

Dendritic spines are the major sites for receiving excitatory synaptic inputs and play important roles in neuronal signal

\footnotetext{
Received July 27, 2020; revised June 5, 2021; accepted June 10, 2021.

Author contributions: Y.S., N.K., and R.Y. designed research; Y.S. performed research; M.S. contributed unpublished reagents/analytic tools; Y.S. and R.Y. analyzed data; Y.S. and R.Y. wrote the paper.

This work was supported by National Institutes of Health Grants R35-NS-116804, DP1-NS-096787, and R01-MH-080047, and the Brain Research Foundation. We thank Jessica Martin for performing some of the initial 3D reconstruction, Jaime Richards for preparing organotypic slice cultures, Takayasu Minuki and Debbie Guerrero-Given for technical assistance, and Lesley Colgan for critical reading. We also thank the Max Planck Florida Institute for Neuroscience Imaging Center for instrument and technical support.

The authors declare no competing financial interests.

Correspondence should be addressed to Ryohei Yasuda at ryohei.yasuda@mpfi.org.

https://doi.org/10.1523/JNEUROSCI.1964-20.2021

Copyright $\odot 2021$ the authors
}

transduction, memory storage, and neuronal circuit organization. Plasticity of spine structure plays a critical role in electrophysiological plasticity, learning, and memory (Sala and Segal, 2014). In particular, it has been reported that activity-dependent spine enlargement [i.e., structural LTP (sLTP)] is correlated with LTP (Lang et al., 2004; Matsuzaki et al., 2004; Kopec et al., 2006) and memory formation (Hayashi-Takagi et al., 2015). Ultrastructural correlates of LTP have also been studied using electron micrographs of LTP-induced brain slices under a condition where a large fraction of spines was electrically stimulated. These studies revealed that LTP is correlated with, on average, a larger spine volume and PSD area, a higher number of concave spine profiles, an increase in perforated PSD, and a higher fraction of polyribosome-containing spines (Van Harreveld and Fifkova, 1975; Desmond and 
Levy, 1986; Harris et al., 1992; Toni et al., 2001; Ostroff et al., 2002). Furthermore, LTP was found to be associated with the generation of multi-innervating spines and multiple spine synapses (Toni et al., 1999; Giese et al., 2015).

However, strong and synchronous LTP stimulation induced in a large population of dendritic spines makes it challenging to differentiate spines that receive direct LTP stimulation from spines that are influenced by surrounding ones. Recent studies have addressed this issue by inducing sLTP and LTP with two-photon (2P) glutamate uncaging in single dendritic spines, and the spines have been visualized under transmission electron microscopy (TEM) by correlating light and electron microscopy (Bosch et al., 2014; Meyer et al., 2014). It has been found that, while the spine volume and synaptic transmission increase rapidly after sLTP induction, the PSD area increases slowly over several hours (Matsuzaki et al., 2004; Bosch et al., 2014; Meyer et al., 2014). The difference in timing implies the reorganization of substructures of synapses during sLTP. However, the throughput of these methods has been low, and thus detailed analyses of PSD morphology have not been done.

In addition to these EM studies, nanometer-scale PSD structure and plasticity have also been studied using optical super-resolution microscopy (MacGillavry et al., 2013; Nair et al., 2013; Hruska et al., 2018; Masch et al., 2018; Wegner et al., 2018). These studies suggest that, in dissociated culture, the synaptic scaffold is made of isolated nanoclusters (MacGillavry et al., 2013; Nair et al., 2013; Hruska et al., 2018), which increase in number during chemically induced LTP (MacGillavry et al., 2013; Hruska et al., 2018). However, a recent in vivo study using stimulated emission depletion microscopy suggested that PSD95 does not form segregated clusters, but nearly half of the postsynaptic scaffolds show complex spatial organization (Masch et al., 2018). Thus, overall, how the PSD and surrounding membrane change their nanometer-level structure during sLTP still remains elusive.

Here, we demonstrate that single spines stimulated and imaged under a 2P microscope can be reliably and efficiently reimaged with automated tape-collecting ultramicrotome (ATUMtome) sectioning and scanning EM (SEM). This technique allows for clear visualization of subspine ultrastructures, including the PSD and synaptic membrane (Kamasawa et al., 2015; Sun et al., 2020). 3D reconstruction of the stimulated spines showed that the PSD underwent rapid and sustained structural changes, including perforation and segmentation, while its size slowly developed. Associated with changes in the PSD structure, we also observed an immediate and sustained expansion of the nonsynaptic axonspine interface (nsASI), which has a narrow extracellular space bordered by axon and spine membrane without PSD. The nsASI expansion, coordinated with the increase in structural complexity of PSD, may support a rapid increase in synaptic transmission during sLTP.

\section{Materials and Methods}

Hippocampal organotypic slice culture and gene gun transfection. All of the animals were handled according to approved Institutional Animal Care and Use Committee protocols of the Max Planck Florida Institute for Neuroscience. Hippocampal organotypic slice cultures were prepared from postnatal day 4-6 mice of either sex, as previously described (Gogolla et al., 2006). After 10-12 d in culture, CAG-EGFP plasmid (catalog \#16664, Addgene) transfection was performed by a Helios gene gun (Woods and Zito, 2008). Two days after transfection, slices with sparsely labeled CA1 pyramidal neurons were selected for glutamate uncaging.

$2 p$ glutamate uncaging. 4-Methoxy-7-nitroindolinyl-caged-L-glutamate (MNI)-glutamate uncaging was performed with $2 \mathrm{P}$ laser microscopy $(720 \mathrm{~nm})$ with $4 \mathrm{~mW}$ power, $6 \mathrm{~ms}$ dwell time, $0.5 \mathrm{~Hz} \times 30$ stimulation for LTP induction at room temperature. The buffer for $2 \mathrm{P}$ imaging and uncaging was $\mathrm{Mg}^{2+}$-free ACSF containing $127 \mathrm{~mm} \mathrm{NaCl}$, $2.5 \mathrm{~mm} \mathrm{KCl}, 25 \mathrm{~mm} \mathrm{NaHCO} 3,1.25 \mathrm{~mm} \mathrm{NaH}_{2} \mathrm{PO}_{4}, 4 \mathrm{~mm} \mathrm{CaCl}_{2}$, and 25 mM D-glucose plus $1 \mu \mathrm{M}$ TTX and $4 \mathrm{~mm}$ MNI-glutamate aerated with $95 \% \mathrm{O}_{2}$ and $5 \% \mathrm{CO}_{2}$ (Hedrick et al., 2016). The slices were kept in ACSF for at least $10 \mathrm{~min}$ before uncaging and during imaging until fixation. Target spines were selected on secondary dendrites of a CA1 pyramidal neuron (Fig. 1A, Extended Data Fig. 1-1A1,A2). Uncaging was performed at $\sim 0.5 \mu \mathrm{m}$ from the tip of the selected spine (Fig. $1 B$, Extended Data Fig. 1-1A3). Zoom 1 ( $z$-stack, $5 \mu \mathrm{m} /$ step, $\left.200 \times 200 \mu \mathrm{m}^{2}\right)$, zoom 5 $\left(z\right.$-stack, $3 \mu \mathrm{m} /$ step, $\left.40 \times 40 \mu \mathrm{m}^{2}\right)$, and zoom $25(z$-stack, $1 \mu \mathrm{m} /$ step, $\left.8 \times 8 \mu \mathrm{m}^{2}\right)$ images of the whole neuron and specific uncaged region were acquired within $5 \mathrm{~min}$ before uncaging with $2 \mathrm{P}$ microscopy, and zoom 25 images of the same region were taken immediately after uncaging to confirm the success of stimulation (Fig. 1C, Extended Data Fig. 1$1 A 4)$. Hippocampal slices were transferred into a fixative buffer $[2 \%$ paraformaldehyde and $2 \%$ glutaraldehyde in $0.1 \mathrm{M}$ phosphate buffer (PB) at $\mathrm{pH} 7.4 \mathrm{l}$ at different time points $(2-3 \mathrm{~min}, 20 \mathrm{~min}, 2 \mathrm{~h}$ ) after uncaging and kept for $1 \mathrm{~h}$. Slices were then washed in $0.1 \mathrm{M} \mathrm{PB}$ three times for a total of $30 \mathrm{~min}$. Zoom 25 images of target spines were taken again to confirm the maintenance of sLTP (Extended Data Fig. 1-1B1).

Laser-burning marks. Some slices were chosen to test additional fiducial marks for correlation. For these slices, burning marks were introduced with a $2 \mathrm{P}$ laser near the target neurons and target secondary dendrites after slice fixation, positioned $>5 \mu \mathrm{m}$ from the target spines. For easy operation, an uncaging laser at $720 \mathrm{~nm}(30 \mathrm{~mW})$ was used in line scan mode to introduce horizontal burning lines while slices kept perfused in $0.1 \mathrm{M} \mathrm{PB}, \mathrm{pH}$ 7.4. The number of line scans needs to be tested for each sample. Successful burning marks show autofluorescence under 2P microscopy (Extended Data Fig. 1-1B2) and can be visualized in the slice embedded in resin under both light microscopy and EM.

Laser burning significantly improved the efficiency of relocating stimulated spines, especially in estimating the $z$-position for target neurons and spines. However, laser burning is not a must for our workflow, and should be avoided if a long-distance tracing of axons is desired since the structures of interest might be disrupted.

Confocal scanning microscopy imaging. Confocal images were taken with a Zeiss LSM780 confocal scanning microscope. GFP ${ }^{+}$neurons were viewed with a laser excitation wavelength of $488 \mathrm{~nm}$. Images were taken in tile mode to cover the whole slice and in $z$-stack mode $(5 \mu \mathrm{m}$ interval) to cover the $z$-axis range of the target neuron (Extended Data Fig. 1-1B3).

Immuno-EM processing and ultra-thin sectioning. Slices were incubated in $50 \mathrm{~mm}$ glycine in $0.1 \mathrm{M}$ PB for $10 \mathrm{~min}$ to block excessive aldehyde sites. Slices were then cryoprotected with 15-30\% sucrose, followed by two freeze-thaw cycles of 1 min liquid nitrogen permeation. After blocking with normal goat serum and fish skin gelatin, the slices were incubated with anti-GFP primary antibody $(0.1 \mu \mathrm{g} / \mathrm{ml}$; catalog \#ab6556, Abcam) for $40 \mathrm{~h}$, followed by nanogold-conjugated secondary antibody (1:100; catalog \#2003, Nanoprobes) for $16 \mathrm{~h}$. Silver enhancement was then performed with the HQ Silver Enhancement Kit (catalog \#2012, Nanoprobes) to increase the visibility of gold particles. Slices were then postfixed with $0.5 \%$ aqueous $\mathrm{OsO}_{4}$ for $40 \mathrm{~min}$ at $4^{\circ} \mathrm{C}$; stained with $1 \%$ aqueous uranyl acetate for $35 \mathrm{~min}$; dehydrated with gradually increased concentrations of ethanol, acetone, and propylene oxidase; and infiltrated by Durcupan ACE (Sigma-Aldrich).

Slices were trimmed down to the region containing the target neuron according to confocal image localization and light microscopy visualization. The final sample size was $\sim 1 \times 1.5 \mathrm{~mm}$. The samples were then sectioned with a $4 \mathrm{~mm}$ Diatome $35^{\circ} \mathrm{knife}$ by ATUMtome (RMC/ Boeckeler Instruments) at a thickness of $50 \mathrm{~nm}$ and were collected onto rolling Kapton tape (Extended Data Fig. 1-1C1). The Kapton tape was then cut and aligned on 4 inch silicon wafers covered with carbon tape. Three 150 mesh copper grids were put onto blank regions of carbon tape as fiducial marks (Extended Data Fig. 1-1C2). A 5-nm-thick layer of carbon was then coated onto the surface of the wafer using a high-vacuum sputter coater (model ACE600, Leica). 

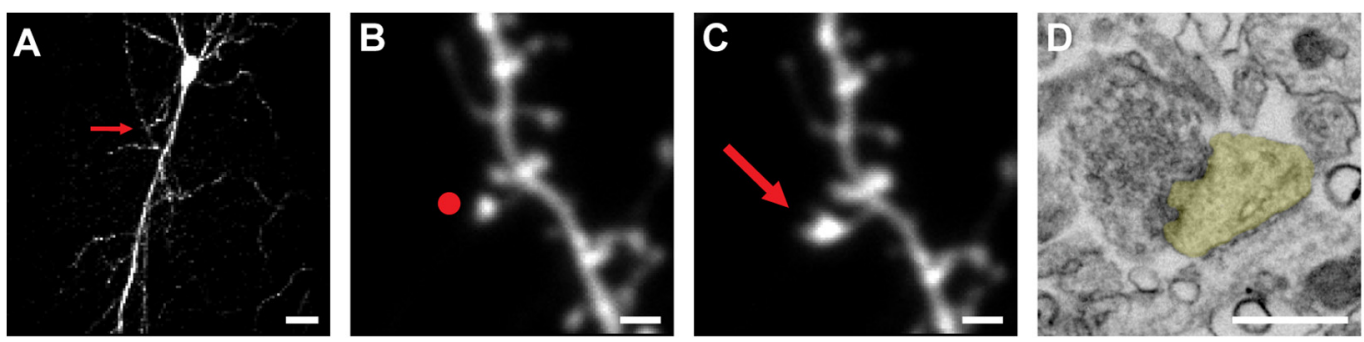

E

G
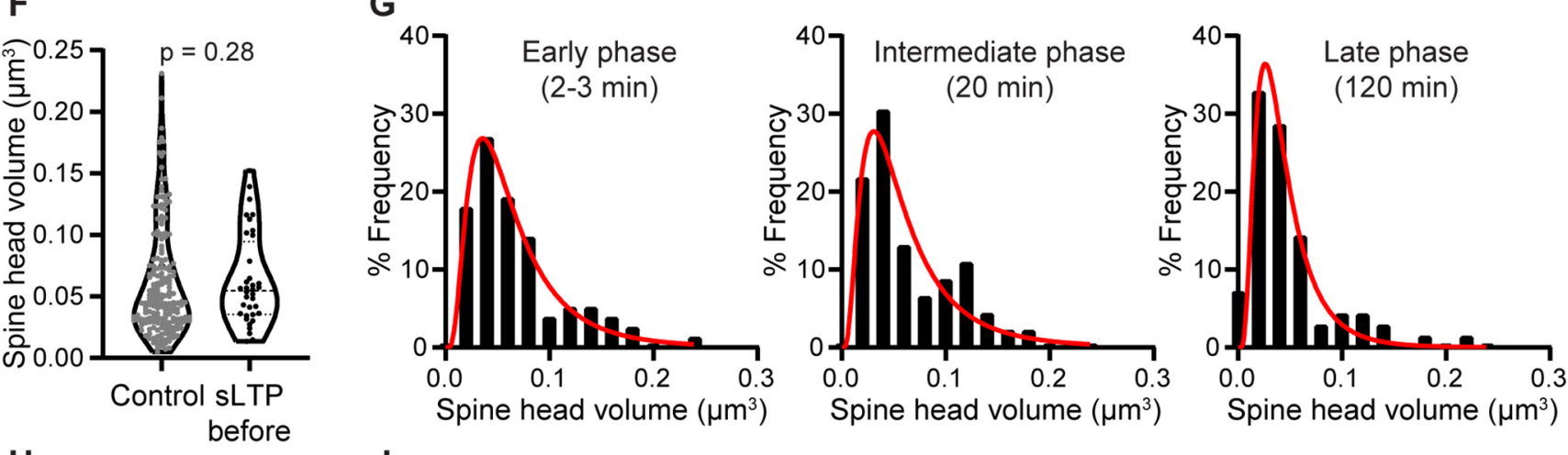

$\mathrm{H}$

$$
\text { I }
$$
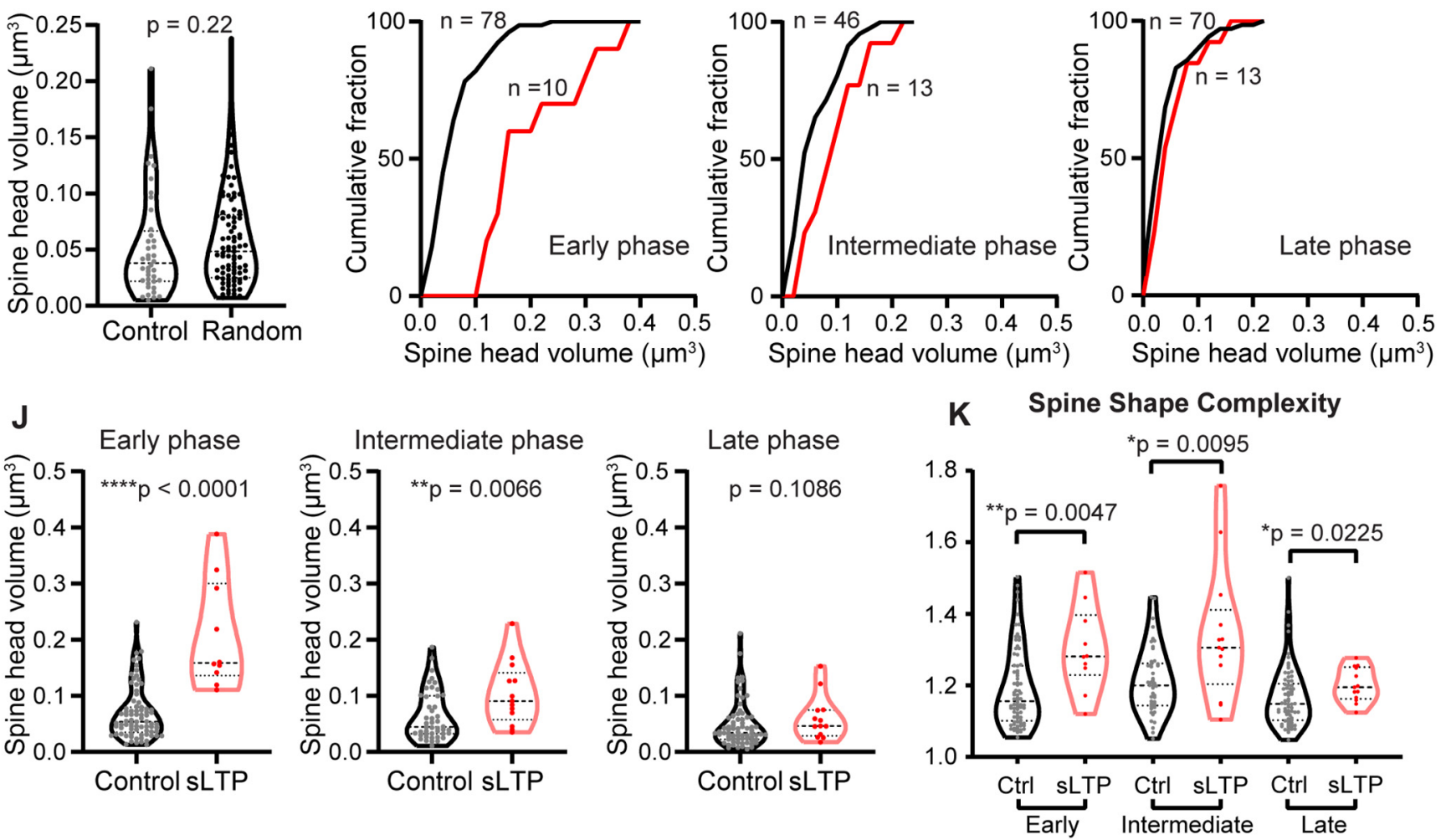

Figure 1. 2P glutamate uncaging at single spine induces spine expansion. $A, 2 \mathrm{P}$ image of an $\mathrm{EGFP}^{+}$(A1 pyramidal neuron in mice organotypic hippocampal slice selected for glutamate uncaging. Red arrow points to the location of target spine. Scale bar, $20 \mu \mathrm{m}$. $\boldsymbol{B}, \boldsymbol{C}, 2 \mathrm{P}$ images of a dendritic spine before $(\boldsymbol{B})$ and after $(\boldsymbol{C}) 2 \mathrm{P}$ glutamate uncaging stimulation. Red dot in $\boldsymbol{B}$ shows the uncaging position, and red arrow in $\boldsymbol{C}$ points to the enlarged dendritic spine. Scale bar, $1 \mu \mathrm{m}$. $\boldsymbol{D}, \mathrm{A} 4 \mathrm{~nm} /$ pixel high-resolution SEM image taken with an InlensDuo detector shows detailed ultrastructure of the modulated dendritic spine in $\boldsymbol{C}$. Spine head is shown in yellow. Scale bar, $0.5 \mu \mathrm{m}$. $\boldsymbol{E}$, 3D reconstruction of the same dendritic spine shown in $\boldsymbol{D}$. Spine head is shown in yellow, and PSD is shown in red. Scale bar, $0.5 \mu \mathrm{m}$. The full workflow for correlative light and electron microscopy is shown in Extended Data Figure 1-1. $\boldsymbol{F}$, The volume of control spines was similar to the volume of sLTP spines before stimulation. Welch's $t$ test was used on log-transformed data. G, Histograms with log-normal distribution fit curves of raw spine head volume for control spines in early, intermediate, and late phases of sLTP. $\boldsymbol{H}$. Spine head volume for control spines and random spines located $>10 \mu \mathrm{m}$ from sLTP spine at late phase of sLTP. Welch's $t$ test was used on log-transformed data. I, Cumulative distribution of raw spine head volume for control and sLTP spines in early, intermediate, and late phases of sLTP. J, Spine head volume for control and sLTP spines in early, intermediate, and late phases of sLTP. Welch's $t$ test was used on log-transformed data. $\boldsymbol{K}$, Spine shape complexity index for control (Ctrl) and SLTP spine at early, intermediate, and late phases of sLTP. Mann-Whitney $t$ test was used. Raw measurements for spine parameters and 3D reconstruction for each spine is shown in Extended Data Figure $1-2 .{ }^{*} p<0.05,{ }^{* *} p<0.01,{ }^{* * *} p<0.0001$. 
SEM imaging. SEM imaging was performed with Zeiss Merlin VP Compact using Zeiss Atlas 5 AT software (version 5.0.49.4). An overview image of the wafer was taken with a digital camera for the alignment of the physical position of the wafer in the SEM chamber (Extended Data Fig. 1-1C2). The alignment was performed by using the grids on the wafer as fiducial marks. Approximate locations of sections could be visualized in the digital camera overview image. Then low-resolution $(2 \mu \mathrm{m} /$ pixel) images of whole sections were first taken to accurately locate the position of the sections by secondary electron (SE2) detector $(2.5-3 \mathrm{kV}$, $0.8 \mu$ s dwell time; Extended Data Fig. 1-1D1). The relative location of the neuron labeled with immunogold in the serial sections was then estimated according to the overview confocal image. A backscattered electron (BSE) detector (BSD4A, $8-10 \mathrm{kV}, 10 \mu$ s dwell time) was used at this step to search for profiles labeled with the silver-enhanced nanogold particles. This was followed by the acquisition of a series of medium-resolution $(30 \mathrm{~nm} /$ pixel) images of the target neuron (Extended Data Fig. 1$1 D 2)$. A rough $3 \mathrm{D}$ reconstruction of the neuron containing its soma and target secondary dendrite regions was made by Amira software (version 2019.3; Thermo Fisher Scientific; Extended Data Fig. 1-1E1). By comparing the shape of the 3D reconstruction of a labeled neuron and the originally captured $2 \mathrm{P}$ microscopy image, the target neuron was confirmed, and the target secondary dendrite was determined. For slices with laserburning marks, the step to reconstruct the whole neuron for the correlation was omitted. Burning marks were easily found in EM as empty straight lines (Extended Data Fig. 1-1D3) and could be used directly for the identification of targeted dendritic segments.

Final high-resolution images $(4 \mathrm{~nm} /$ pixel) were then taken using an In-lens Duo Detector in BSE mode $(2.5 \mathrm{kV}, 75 \mu$ s dwell time) or an aBSD detector $(8-10 \mathrm{kV}, 25-40 \mu \mathrm{s}$ dwell time) to visualize ultrastructure of the target spines and their surrounding regions (Fig. $1 D$, Extended Data Fig. 1-1D4). Target spines were identified by comparing the spine shape and location between $3 \mathrm{D}$ reconstructed dendritic segments and $2 \mathrm{P}$ images. The ultrastructure required for analyzing spines and synapses, such as presynaptic and postsynaptic membranes, synaptic cleft, and PSD, is clearly visualized. 3D reconstruction of dendritic spines was performed in Amira (Fig. 1E, Extended Data Fig. 1-1E2).

In this study, the success rate for collecting final high-resolution images for the target spines was 44\% (15 of 34 slices). The reasons for failed experiments include the following: (1) poor ultrastructure preservation; (2) weak immunoreaction to locate target neuron under a dissecting microscope; (3) heavy silver enhancement causing large deposition of nanogold particles disturbed the ultrastructure observation; (4) nonoptimal conditions of the Kapton tape (having scratches or debris on its surface) and/or ultra-thin sections (having wrinkles, chatters, or damages) that interrupted target spine imaging; and (5) accident loss of the region of interest in the trimming process. The success rate significantly increased with experience and improved quality control on slice health and Kapton tape.

Estimation of spine volume before $2 P$ glutamate uncaging. SLTP spines were imaged in $2 \mathrm{P}$ microscopy within $5 \mathrm{~min}$ before glutamate uncaging and after chemical fixation. The spine volume increase was quantified as a change in GFP fluorescence intensity. The spine volume after fixation was calculated from $3 \mathrm{D}$ reconstruction of the spines with EM images, and the spine volume before uncaging was calculated as the spine volume measured in EM divided by the volume change measured optically.

Image analysis. ASI was traced as the postsynaptic part of the interface between the spine head and the opposing presynaptic bouton. PSD was identified as the electron-dense region in ASI, and nsASI was identified as the ASI membrane without PSD.

Spine shape complexity and PSD complexity. Dendritic spines and PSDs were reconstructed in $3 \mathrm{D}$ in Amira, then exported into the MATLAB format to create a shadowed PSD region on the spine surface. Spine head volume and surface area were measured after reconstruction and were used to calculate the spine shape complexity index with the following formula, with which a sphere-shaped spine head generates an index equal to 1 , as follows:
Spine shape complexity index $=\frac{(\text { Spine surface area } / 4 \pi)^{1 / 2}}{(3 \text { Spine head volume } / 4 \pi)^{1 / 3}}$.

The smoothed PSD area and perimeter were measured and used to calculate the complexity index with the following formula, with which a circle-shaped PSD generates an index equal to 1, as follows:

$$
\text { PSD complexity index }=\frac{(\text { PSD perimeter })^{2}}{4 \pi(\text { PSD area })} .
$$

Experimental design and statistical analyses. We introduced singlespine sLTP using $2 \mathrm{P}$ glutamate uncaging in hippocampal organotypic slice culture because of their easy operation and stability to produce good ultrastructure preservation. We imaged $\sim 100$ sections around the sLTP spines. All spines located on the same secondary dendrite with sLTP spines were used as control spines, except those with PSD parallel to the section plane. Based on the dendrite orientation, 1-31 control spines were taken for each sLTP spine, and control spines were all located within $20 \mu \mathrm{m}$ from the sLTP spine. Since we compare reconstructed dendrites with $2 \mathrm{P}$ images to ensure correct correlation, it is not feasible to make the spine ultrastructure measurement process blind.

Statistical tests were performed in GraphPad Prism 6. Measured ultrastructural data were first tested for normal distribution and log-normal distribution with a D'Agostino-Pearson test. Data without skewness were compared with Welch's $t$ test and shown with the mean and SEM for each control versus sLTP group. Data without skewness after logtransformation (consistent with log-normal distribution) were log transformed and compared with the Welch's test. For data that did not follow normal distribution or log-normal distribution, a Mann-Whitney test was performed for comparison. To compare the growth of PSD, ASI, and nsASI with spine volume growth, their ratio was analyzed with twoway ANOVA. Data consistent with log-normal distribution were log transformed before two-way ANOVA. A post hoc Tukey's multiple-comparison test was performed to compare among different sLTP phases, and a post hoc Bonferroni's multiple-comparison test was performed to compare between control and sLTP conditions. An extra sum-of-squares $F$ test was used to compare regression lines. All statistics are summarized in Tables 1 and 2. Raw data are available in Extended Data Figure 1-2.

\section{Results \\ Correlating dendritic spines imaged by $2 \mathrm{P}$ microscopy and SEM}

We established an efficient method to analyze the 3D ultrastructure of spines undergoing spine-specific sLTP in organotypic hippocampal slices of mice. Spine structural plasticity was first imaged in $2 \mathrm{P}$ microscopy and then identified in EM by combining pre-embedding immunolabeling of GFP with ATUMtome serial sectioning and SEM imaging (Fig. $1 A-E$, Extended Data Fig. 1-1, whole flow). We induced sLTP at single spines with glutamate uncaging by delivering $2 \mathrm{P}$ laser pulses located $\sim 0.5 \mu \mathrm{m}$ from the tip of the spine head in GFP-expressing CA1 pyramidal neurons in organotypic hippocampal slices (Fig. $1 A-C$, Extended Data Fig. 1-1A; Matsuzaki et al., 2001, 2004; Harvey et al., 2008). These slices were then fixed at different time points after uncaging stimulation to capture the structural changes at the following different SLTP phases: early phase (2-3 min), intermediate phase (20 min), and late phase (120 min; Extended Data Fig. 1-1B1). GFP expression in entire slices was imaged in confocal microscopy, and optional burning marks were introduced near the target dendritic segment with $2 \mathrm{P}$ lasers to facilitate EM correlation (Extended Data Fig. 1-1B2,B3). After immunostaining of GFP with nanogold particles, we performed silver enhancement, heavy metal staining, dehydration, and resin embedding. Using ATUMtome, we cut and collected ultra-thin sections $(50 \mathrm{~nm})$, which were imaged in SEM at low resolution $(2 \mu \mathrm{m} /$ pixel $)$ and 
Table 1. Comparison of the ultrastructures of dendritic spines in control and sLTP conditions at early, intermediate and late phases of sLTP

\begin{tabular}{|c|c|c|c|c|c|c|}
\hline \multirow[b]{4}{*}{ Feature } & \multicolumn{2}{|l|}{ Early } & \multicolumn{2}{|l|}{ Intermediate } & \multicolumn{2}{|l|}{ Late } \\
\hline & \multicolumn{2}{|c|}{7 neurons from 3 batches of slices ${ }^{a}$} & \multicolumn{2}{|c|}{7 neurons from 5 batches of slices } & \multicolumn{2}{|c|}{4 neurons from 3 batches of slices } \\
\hline & Control, $n=78$ & sLTP, $n=10$ & Control, $n=46$ & sLTP, $n=13$ & Control, $n=70$ & sLTP, $n=13$ \\
\hline & Median (IQR) Mean \pm SD & Median (IQR) Mean \pm SD & Median (IQR) Mean \pm SD & Median (IQR) Mean \pm SD & Median (IQR) Mean \pm SD & Median (IQR) Mean \pm SD \\
\hline \multirow[t]{3}{*}{ Spine head volume $\left(\mu \mathrm{m}^{3}\right)^{b}$} & $0.05379(0.03476-0.07978)$ & $0.1583(0.136-0.2999)$ & $0.04459(0.03233-0.09994)$ & $0.0904(0.05757-0.141)$ & $0.03414(0.02254-0.06087)$ & $0.0461(0.02846-0.07454)$ \\
\hline & $0.06828 \pm 0.04576$ & $0.2066 \pm 0.0959$ & $0.06393 \pm 0.04349$ & $0.1035 \pm 0.0563$ & $0.04819 \pm 0.04045$ & $0.0596 \pm 0.0392$ \\
\hline & $p<0.0001$ & & $p=0.0066$ & & $p=0.1086$ & \\
\hline \multirow[t]{3}{*}{ Spine surface $\left(\mu \mathrm{m}^{2}\right)^{b}$} & $0.9552(0.6714-1.422)$ & $2.88(2.149-3.346)$ & $0.9292(0.6413-1.601)$ & $1.751(1.282-2.169)$ & $0.6944(0.4661-1.072)$ & $0.8752(0.6654-1.289)$ \\
\hline & $1.147 \pm 0.6790$ & $2.784 \pm 0.7806$ & $1.13 \pm 0.6409$ & $1.895 \pm 0.9968$ & $0.8520 \pm 0.5668$ & $1.03 \pm 0.4711$ \\
\hline & $p<0.0001$ & & $p=0.0012$ & & $p=0.0572$ & \\
\hline \multirow[t]{3}{*}{$\operatorname{PSD}\left(\mu \mathrm{m}^{2}\right)^{b}$} & $0.112(0.07733-0.1754)$ & $0.1856(0.1486-0.246)$ & $0.07884(0.05070-0.1571)$ & $0.103(0.06171-0.1361)$ & $0.07141(0.04262-0.09763)$ & $0.09868(0.04199-0.1253)$ \\
\hline & $0.1345 \pm 0.08413$ & $0.1971 \pm 0.08314$ & $0.1120 \pm 0.09041$ & $0.09983 \pm 0.04134$ & $0.08631 \pm 0.07030$ & $0.116 \pm 0.09073$ \\
\hline & $p=0.0167$ & & $p=0.7213$ & & $p=0.1805$ & \\
\hline \multirow[t]{3}{*}{ ASI $\left(\mu \mathrm{m}^{2}\right)^{b}$} & $0.1895(0.1214-0.3368)$ & $0.4183(0.3209-0.4444)$ & $0.1730(0.1036-0.2750)$ & $0.244(0.1975-0.3612)$ & $0.1248(0.07355-0.1871)$ & $0.2047(0.1504-0.259)$ \\
\hline & $0.2348 \pm 0.1632$ & $0.3845 \pm 0.1307$ & $0.2166 \pm 0.1525$ & $0.2749 \pm 0.1161$ & $0.1691 \pm 0.1655$ & $0.2429 \pm 0.1561$ \\
\hline & $p=0.0014$ & & $p=0.0552$ & & $p=0.0215$ & \\
\hline \multirow[t]{3}{*}{ nsASI $\left(\mu \mathrm{m}^{2}\right)^{b}$} & $0.06453(0.03304-0.1442)$ & $0.1975(0.1527-0.2427)$ & $0.08161(0.04831-0.1303)$ & $0.1667(0.1144-0.2273)$ & $0.04809(0.02426-0.1054)$ & $0.1297(0.08588-0.1543)$ \\
\hline & $0.1004 \pm 0.09104$ & $0.1874 \pm 0.06053$ & $0.1054 \pm 0.0775$ & $0.175 \pm 0.09462$ & $0.08278 \pm 0.1103$ & $0.1269 \pm 0.07147$ \\
\hline & $p<0.0001$ & & $p=0.0132$ & & $p=0.0029$ & \\
\hline
\end{tabular}

IQR, Interquartile range.

${ }^{a}$ One neuron per slice. One batch of slices is from one litter of pups.

${ }^{b}$ Data are consistent with log-normal distribution. Welch's $t$ test was performed on log-transformed data.

Table 2. Comparison of the relative changes of dendritic spine ultrastructures in control and sLTP conditions at early, intermediate, and late phases of sLTP

\begin{tabular}{|c|c|c|c|c|c|}
\hline & & & PSD/spine volume ${ }^{a}$ & ASI/spine volume $^{a}$ & nsASI / spine volume ${ }^{6}$ \\
\hline \multirow[t]{3}{*}{$\begin{array}{l}\text { Two-way ANOVA } \\
\text { Source of variation }\end{array}$} & \multicolumn{2}{|c|}{ Interaction } & $\begin{array}{l}F_{(2,224)}=5.261 \\
* * 0.0059\end{array}$ & $\begin{array}{l}F_{(2,224)}=7.123 \\
* * p=0.0010\end{array}$ & $\begin{array}{l}F_{(2,224)}=3.083 \\
{ }^{*} p=0.0478\end{array}$ \\
\hline & \multicolumn{2}{|c|}{ Phase (early/intermediate/late) } & $\begin{array}{l}F_{(2,224)}=5.579 \\
*^{*} p=0.0043\end{array}$ & $\begin{array}{l}F_{(2,224)}=7.809 \\
* * * p=0.0005\end{array}$ & $\begin{array}{l}F_{(2,224)}=5.475 \\
* * p=0.0048\end{array}$ \\
\hline & \multicolumn{2}{|c|}{ Condition (control/LTP) } & $\begin{array}{l}F_{(1,224)}=23.90 \\
* * * * 00.0001\end{array}$ & $\begin{array}{l}F_{(1,224)}=3.378 \\
p=0.0674\end{array}$ & $\begin{array}{l}F_{(1,224)}=0.541 \\
p=0.4628\end{array}$ \\
\hline \multirow[t]{6}{*}{ Tukey's multiple-comparisons test for phases } & \multirow[t]{3}{*}{ Control } & Early vs intermediate & ${ }^{*} p=0.0449$ & $p=0.9829$ & $p=0.0525$ \\
\hline & & Early vs late & $p=0.4387$ & $p=0.9643$ & $p=0.7308$ \\
\hline & & Intermediate vs late & $p=0.4027$ & $p=0.9986$ & $p=0.2324$ \\
\hline & \multirow[t]{3}{*}{ LTP } & Early vs intermediate & $p=0.9860$ & $p=0.1981$ & $p=0.1038$ \\
\hline & & Early vs late & ${ }^{*} p=0.0108$ & ${ }^{* * *} p=0.0002$ & ${ }^{*} p=0.011$ \\
\hline & & Intermediate vs late & ${ }^{*} p=0.0096$ & ${ }^{*} p=0.0309$ & $p=0.6218$ \\
\hline \multirow[t]{3}{*}{ Bonferroni's multiple-comparisons test on condition } & \multicolumn{2}{|l|}{ Early } & ${ }^{* * * *} p<0.0001$ & ${ }^{* *} p=0.0027$ & $p=0.8004$ \\
\hline & \multicolumn{2}{|c|}{ Intermediate } & ${ }^{* *} p=0.0036$ & $p=0.4996$ & $p>0.9999$ \\
\hline & \multicolumn{2}{|l|}{ Late } & $p>0.9999$ & $p=0.2046$ & $p=0.0506$ \\
\hline
\end{tabular}

${ }^{a}$ Data consistent with Gaussian distribution, two-way ANOVA performed directly.

${ }^{b}$ Data consistent with log-normal distribution, two-way ANOVA performed on log-transformed data. ${ }^{*} p<0.05,{ }^{* *} p<0.01,{ }^{* * *} p<0.001,{ }^{* * * *} p<0.0001$.

intermediate resolution $(30 \mathrm{~nm} /$ pixel; Extended Data Fig. 1-1C, $D 1, D 2)$. Burning marks facilitated the correlation as they can be easily visualized in EM as empty space (Extended Data Fig. 1$1 D 3)$. A rough $3 \mathrm{D}$ reconstruction of the nanogold-positive profile was used for comparison with $2 \mathrm{P}$ neuronal images to identify target neurons (Extended Data Fig. 1-1E1). Following the identification of target neurons and secondary dendrites, we imaged spines that underwent sLTP (sLTP spine) and multiple spines located within the imaging range $(\sim 100$ sections $)$ on the same dendrite (control spine) with high resolution $(4 \mathrm{~nm} /$ pixel; Fig. 1D, Extended Data Fig. 1-1D4). 3D morphology of dendritic spines and PSDs were reconstructed manually from the sections and subjected to further analyses (Fig. 1E, Extended Data Fig. 1$1 E 2)$.

The mean \pm SEM volume of control spines was $0.060 \pm$ $0.003 \mu^{3}(n=194)$, which is consistent with previous studies (Harris et al., 1992). From fluorescence changes following sLTP and the volume of sLTP spines in EM, we estimated the average volume of sLTP spines before the stimulation. The estimated volume (mean \pm SEM, $0.063 \pm 0.006 \mu \mathrm{m}^{3} ; n=36$ ) was similar to the volume of control spines, suggesting that our sLTP spines represent average spines in terms of their volume before sLTP (Fig. 1F, Extended Data Fig. 1-2). We found that control spine volume distribution was skewed from the normal distribution and can be expressed by log-normal function (Fig. 1G; Loewenstein et al., 2011). Thus, for the rest of the study, we performed parametric statistics after log transformation for datasets consistent with a log-normal distribution. Spine volume in control spines in the late phase was significantly smaller than that in the early phase (ANOVA on log-transformed data, $p=0.002$ ). However, since the same trend was also observed in spines on different neurons located away from the stimulated spines $(>10 \mu \mathrm{m}$; Fig. $1 \mathrm{H})$, this phenomenon is likely because of the prolonged incubation of slices in ACSF containing MNI-glutamate and TTX, instead of the heterosynaptic LTD (Harvey and Svoboda, 2007; Oh et al., 2015; Hedrick et al., 2016). For such reasons, we compared only sLTP spines and surrounding spines fixed at the same time point. As expected, sLTP spines showed a 


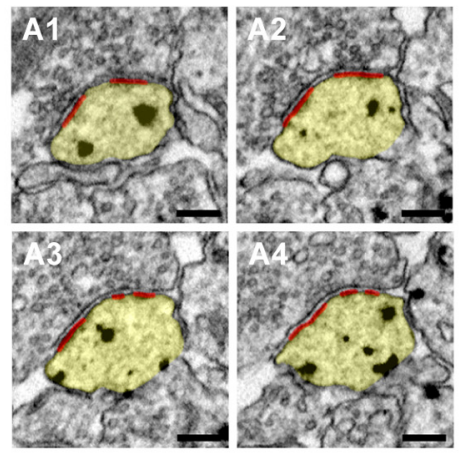

B

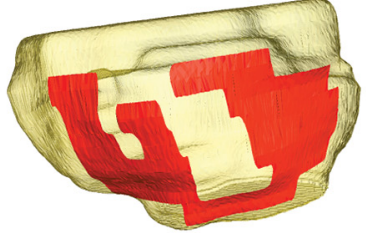

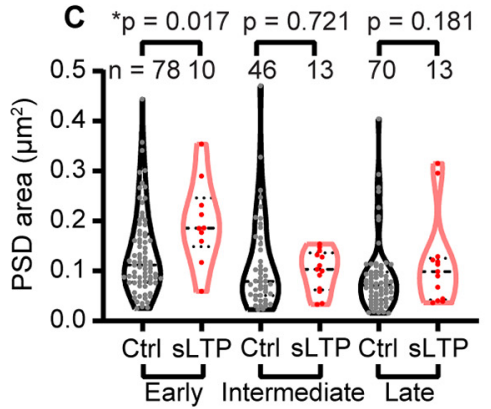
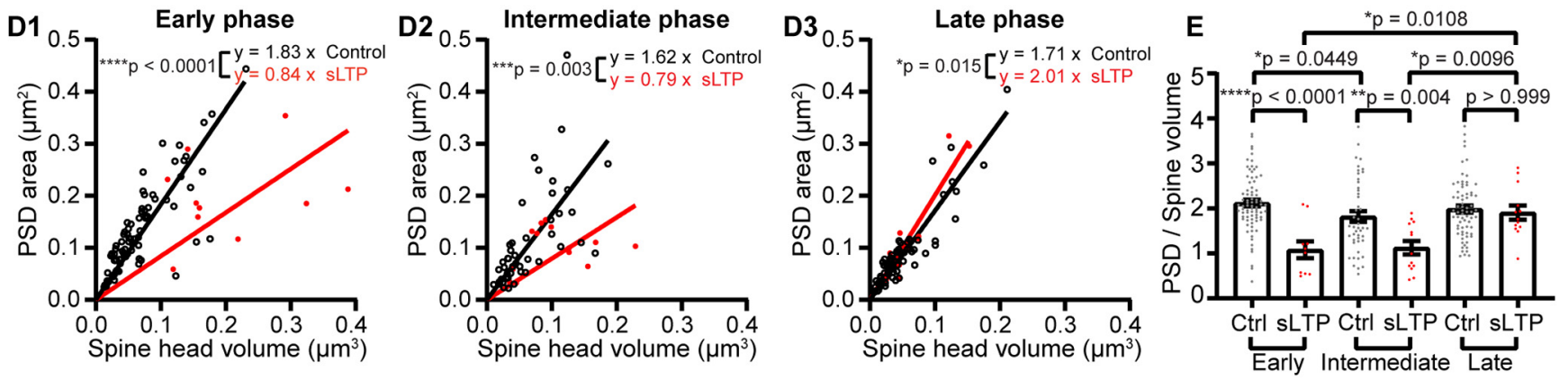

Figure 2. SLTP induction disrupts the correlation between PSD and spine head volume. $\boldsymbol{A}, \boldsymbol{B}$, Serial images of an example spine $(\boldsymbol{A})$ and $3 D$ reconstruction of the example spine $(\boldsymbol{B})$, with spine head shown in yellow and PSD shown in red. Scale bar, $200 \mathrm{~nm}$. C, PSD size at early, intermediate, and late phases of sLTP. Welch's $t$ test on log-transformed data. $D$, Correlation between spine head volume and PSD size for control spines (Ctrl) and SLTP spines at early, intermediate, and late phases of sLTP. Regression line forced through origin. Black line and open circles represent control spines, and red line and solid dots represent sLTP spines at each phase of sLTP. The $p$ value was calculated by comparing the two regression lines with an extra sum-of-squares $F$ test. $\boldsymbol{E}$, Ratio of PSD area to spine volume at early, intermediate, and late phases of sLTP. Two-way ANOVA followed by Bonferroni's post hoc test was used for comparison between control and sLTP spines, and Tukey's post hoc test was used for comparison among different sLTP phases. ${ }^{*} p<0.05,{ }^{* *} p<0.01,{ }^{* * *} p<0.001,{ }^{* * * *} p<0.0001$.

significantly larger size during the early and intermediate phases compared with control spines. At the late phase, the volume of sLTP spines was larger than that before stimulation (mean \pm SEM fold change, 1.33 \pm 0.06 ; Extended Data Fig. 1-2). However, because of the large variability in control spine volume and the relatively small increases in sLTP spine volume at the late phase, the spine volume was not statistically different between the control and sLTP groups (Fig. 1I,J, Table 1). Similarly, we found that the spine surface area increased after sLTP induction during early and intermediate phases, but not in the late phase (Table 1). Furthermore, the shape of the spine head became dramatically more complicated after sLTP induction. To quantify the spine shape complexity, we defined the spine shape complexity index as the ratio of the square root of the spine surface to the cube root of the spine volume, normalized so that a sphere spine has an index equal to 1 . The index was significantly higher in sLTP spines at all phases (Fig. $1 K$ ).

\section{PSD reorganization after $S L T P$ induction}

We next reconstructed the PSD in 3D for all of the spines serially imaged to further characterize their structural features (Fig. $2 \mathrm{~A}$, $B)$. The PSD area size in sLTP spines was significantly larger than that in control spines at the early phase of sLTP (Fig. 2C, Table 1), albeit to a lower degree than the spine volume change (Fig. $2 D, E$ ). In control spines, the PSD area correlated tightly with the spine volume (Fig. $2 D$ ), which is consistent with previous studies (Harris and Stevens, 1989; Bosch et al., 2014). sLTP induction broke the correlation between PSD size and spine volume. The ratio of the PSD size to spine head volume dramatically decreased at the early and intermediate phases, suggesting that the PSD growth was much smaller than the spine volume changes during these phases. The slope of regression lines of
PSD size on spine volume at the late phase for sLTP spines was higher than that for control spines (Fig. 2D3). To compare PSD area and spine volume growth, we analyzed the ratio of PSD area to spine volume at different time points (Fig. 2E). Two-way ANOVA for stimulation conditions (control and sLTP) and time points (early, intermediate, and late) showed that the datasets were significantly different between control and sLTP spines $(p<0.0001)$, and also over time points $(p=0.0043)$, with significant interaction between stimulation conditions and time points $(p=0.0059)$. Post hoc multiple-comparison analyses showed that sLTP spines had significantly smaller PSD-to-spine volume ratios in the early phase $(p<0.0001)$ and intermediate phase $(p=0.0036)$, but not in the late phase $(p=1.0)$. Furthermore, sLTP spines had a significant increase in the ratio in the late phase, compared with the early phase $(p=0.0108)$ and intermediate phase ( $p=0.0096$; Table 2$)$. Thus, these results suggest that PSD growth is slower than spine volume growth by approximately hours, which is consistent with previous studies (Bosch et al., 2014; Meyer et al., 2014).

We further analyzed the detailed nanometer-scale structure of the PSD (Fig. $3 A-H$ ). We observed PSDs with simple, fenestrated, horseshoe, and segmented shapes, as previously reported (Fig. 3A-D). In addition, we also observed PSDs with irregular shapes that did not fit previous categories (Fig. $3 E, F$ ) and with shapes including multiple features (Fig. 3G, horseshoe and fenestration, $H$, segmentation and fenestration). At all of the time points, a higher percentage of sLTP spines contained PSDs with complex structural features, including fenestration, horseshoe shape, and segmentation, compared with control spines (Fig. 3I). In the early phase, nearly half of the stimulated spines contained segmented PSD, composed of either two or three fragments (Fig. 3J). To quantitatively evaluate the complexity of PSD shape, we calculated a complexity index, defined as the ratio of the square 
A

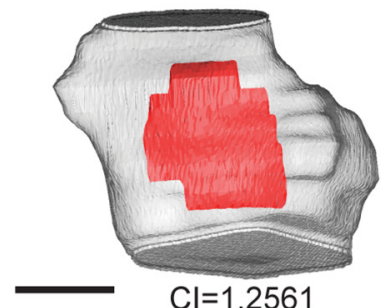

E

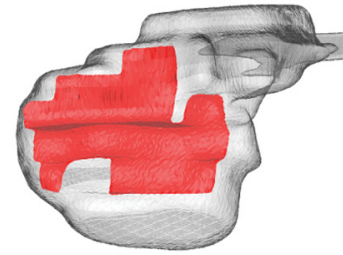

$\mathrm{Cl}=2.0463$

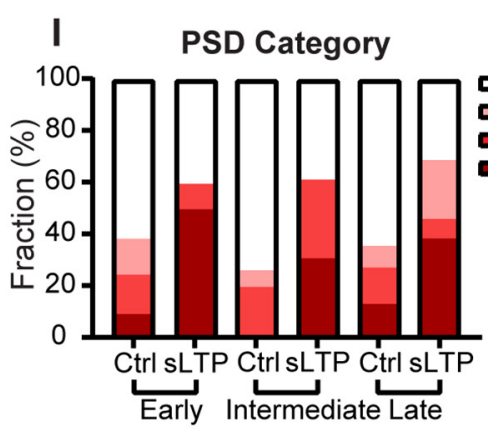

J

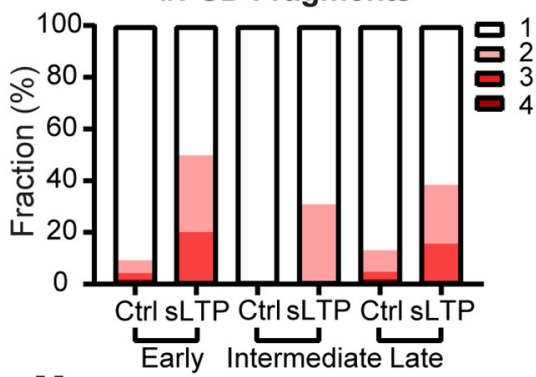

M PSD Shape Complexity

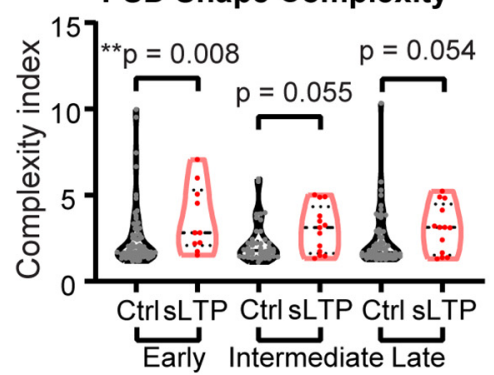

B

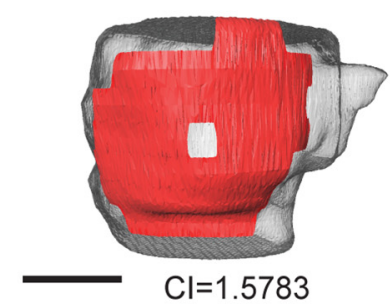

F

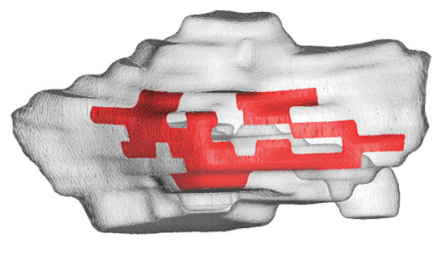

$\mathrm{Cl}=7.9272$
C

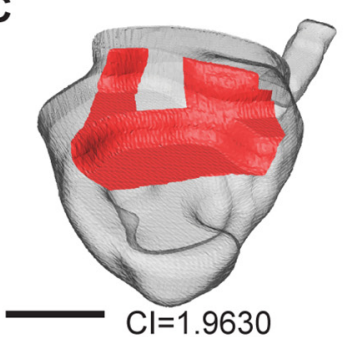

G

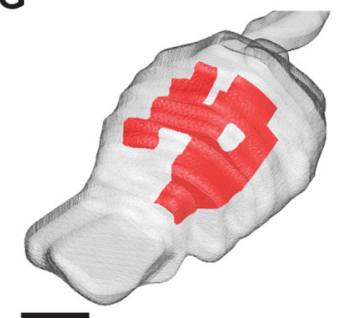

$\mathrm{Cl}=3.2673$

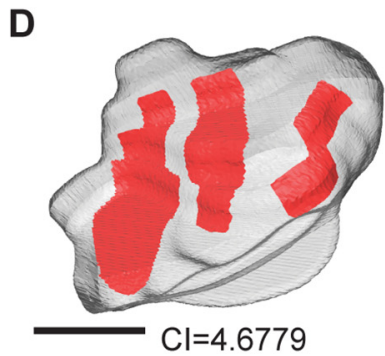

H

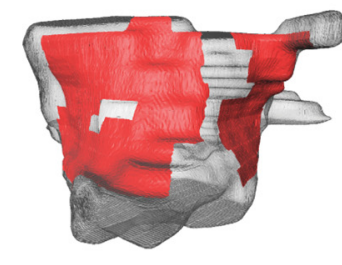

$\mathrm{Cl}=4.9905$
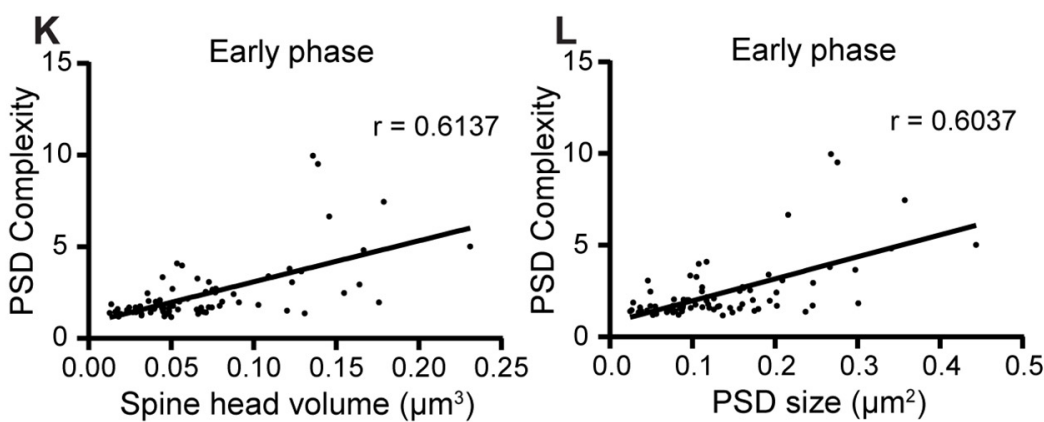

simple

fenestration

horseshoe

segmentation

Spine head volume $\left(\mu \mathrm{m}^{3}\right)$
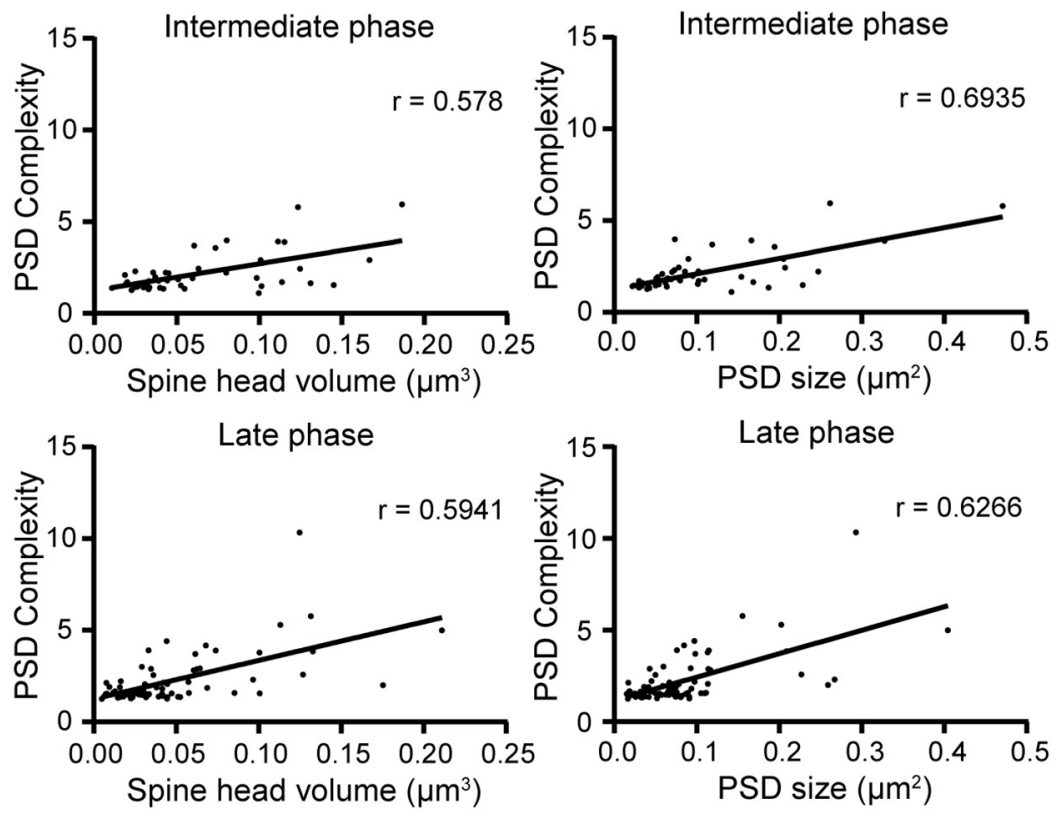

Figure 3. SLTP induces increased PSD complexity. $\boldsymbol{A}-\boldsymbol{H}, 3 \mathrm{D}$ reconstruction of example spines (gray) containing PSD (red) with different complexity indexes (Cls) and different shape categories: simple $(\boldsymbol{A})$, fenestration $(\boldsymbol{B})$, horseshoe $(\boldsymbol{C})$, segmentation $(\boldsymbol{D})$, irregular horseshoe $(\boldsymbol{E}, \boldsymbol{F})$, and combined shape categories $(\boldsymbol{G}, \boldsymbol{H})$. Scale bar, $200 \mathrm{~nm}$. PSD $\mathrm{Cl}$ is shown for individual spine. $I$, Percentage of spines contains PSD in simple, fenestrated, horseshoe, and segmented categories at early, intermediate, and late phases of sLTP in sLTP and control (Ctrl) spines. J, Percentage of spines containing one to four PSD segments at early, intermediate, and late phases of sLTP. $K$, $\boldsymbol{L}$, PSD shape complexity is positively correlated with spine head volume $(\boldsymbol{K})$ and PSD size $(\boldsymbol{L})$ in control spines at all phases of sLTP. $r$, Pearson's correlation coefficient. $\boldsymbol{M}$, PSD complexity index at early, intermediate, and late phases of sLTP. Mann-Whitney $t$ test was used. ${ }^{* *} p<0.01$. 
A \#Innervation

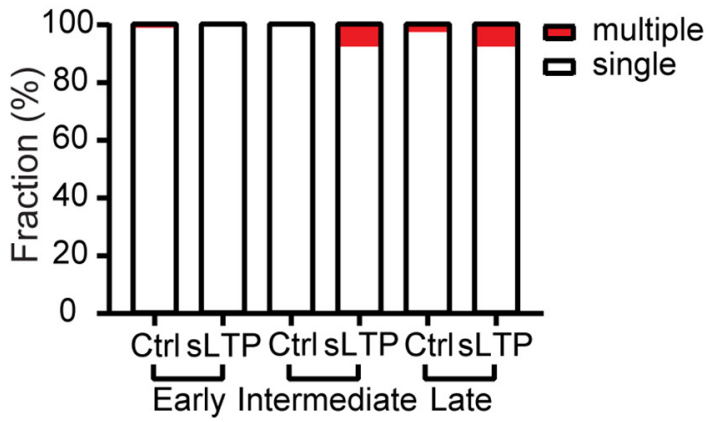

B

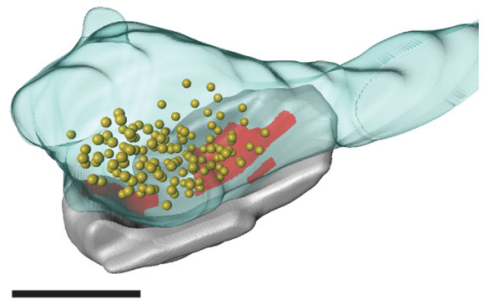

C

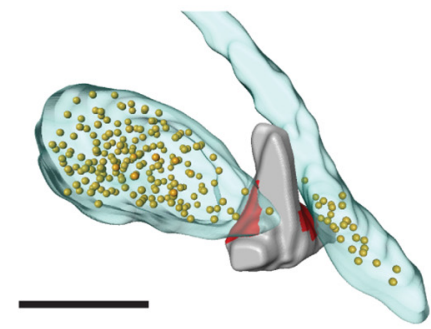

Figure 4. Observation of multi-innervated spines. $A$, Percentage of single- and multi-innervated spines at early, intermediate, and late phases of sLTP for sLTP and control (Ctrl) spines. B, C, 3D reconstruction of example single-innervated spine $(\boldsymbol{B})$ and multi-innervated spine $(\boldsymbol{C})$ with opposing presynaptic boutons and synaptic vesicles. Spines are shown in gray, PSD is shown in red, presynaptic bouton is shown in transparent blue, and synaptic vesicles are shown as golden balls. Scale bar, $500 \mathrm{~nm}$.

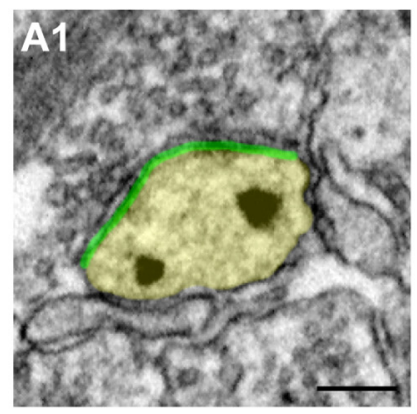

B

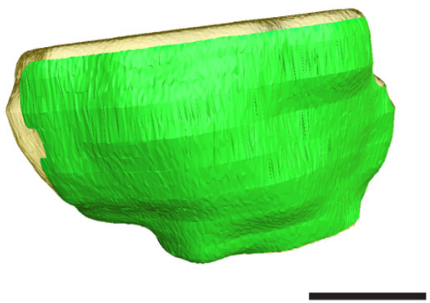

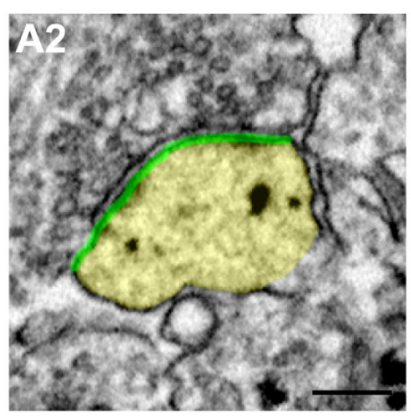

C

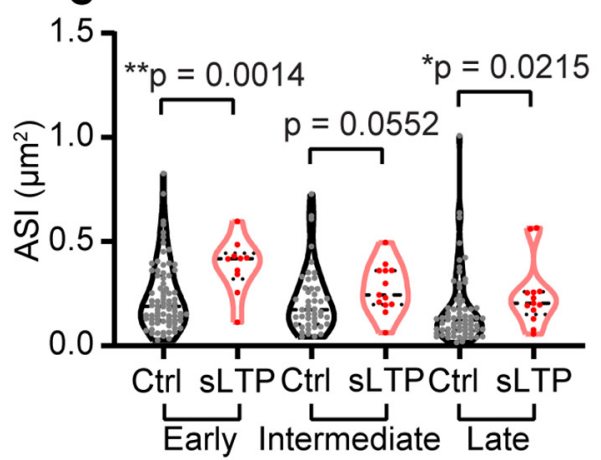

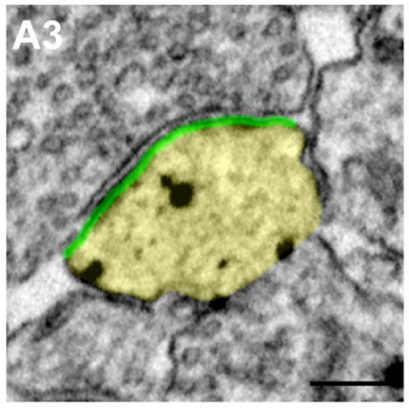

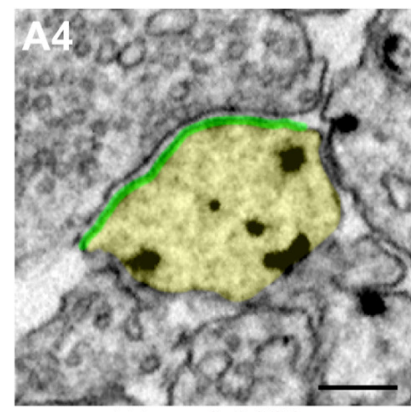

$* * * p=0.0002$

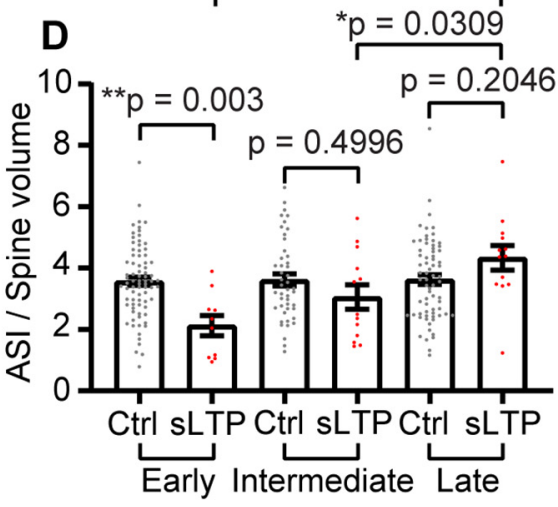

Figure 5. SLTP induces expansion of total ASI postsynaptic membrane. $\boldsymbol{A}, \boldsymbol{B}$, Serial images of an example spine $(\boldsymbol{A})$ and $3 \mathrm{D}$ reconstruction of the example spine $(\boldsymbol{B})$ with spine head shown in yellow and ASI shown in green. Scale bar, $200 \mathrm{~nm}$. C, ASI area size at early, intermediate, and late phases of sLTP for SLTP and control (Ctrl) spines. Welch's $t$ test was used on log-transformed data. $\boldsymbol{D}$, Ratio of ASI area to spine volume at early, intermediate, and late phases of sLTP. Two-way ANOVA followed by Bonferroni's post hoc test was used for comparison between control and sLTP spines, and Tukey's post hoc test was used for comparison among different phases. ${ }^{*} p<0.05$, ${ }^{* *} p<0.01,{ }^{* * *} p<0.001$.

of the perimeter to the area, and normalized so that the index for a circular disk shape equals 1 . We found that a higher PSD complexity index was associated with larger spine volume and PSD area (Fig. $3 K, L$ ). Because of this correlation, the complexity index was highly variable among different spines. We observed that the PSD shape was significantly more complex in sLTP spines than in control spines at the early phase and showed a trend toward higher complexity at the intermediate and late phases of sLTP (Fig. 3M). Overall, our results indicate that a dramatic PSD shape transformation happened immediately after sLTP induction. We also observed that two sLTP spines with segmented PSDs were multiply innervated (intermediate phase, 1 of 13; late phase, 1 of 13), while three control spines were multiply innervated (early phase, 1 of 80; late phase, 2 of 73; Fig. 4).

\section{Nonsynaptic axon-spine interface expansion after sLTP induction}

ASI is the area with thin extracellular space bordered by axon and spine membrane (Fig. 5A,B). A rapid and maintained expansion of ASI was observed after sLTP induction (Fig. $5 C$ ). To quantify the time course of ASI growth compared with spine volume increase, we calculated the ratio of ASI area to spine volume using two-way ANOVA and a post hoc multiple comparison. We found that the ratio was significantly smaller for sLTP in the early phase $(p=0.003)$, but not in the intermediate phase $(p=0.50)$ and late phase $(p=0.20)$. Furthermore, in sLTP spines, the ratio is significantly larger in the late phase compared with early phase $(p=0.0002)$ and intermediate phase ( $p=0.031$; Fig. $5 D$, Table 2$)$. Thus, the results suggest that ASI growth is slower than spine volume growth, and the ratio changes progressively toward the control level. 

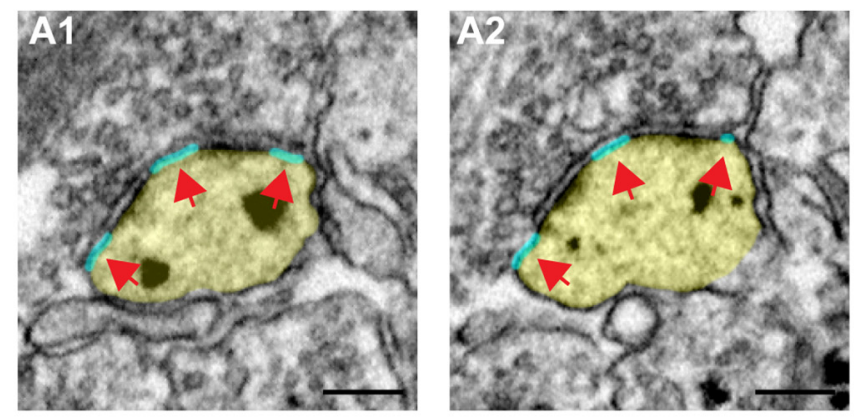

B

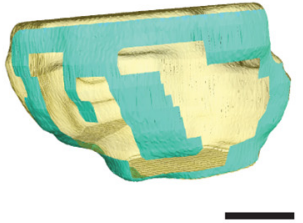

C
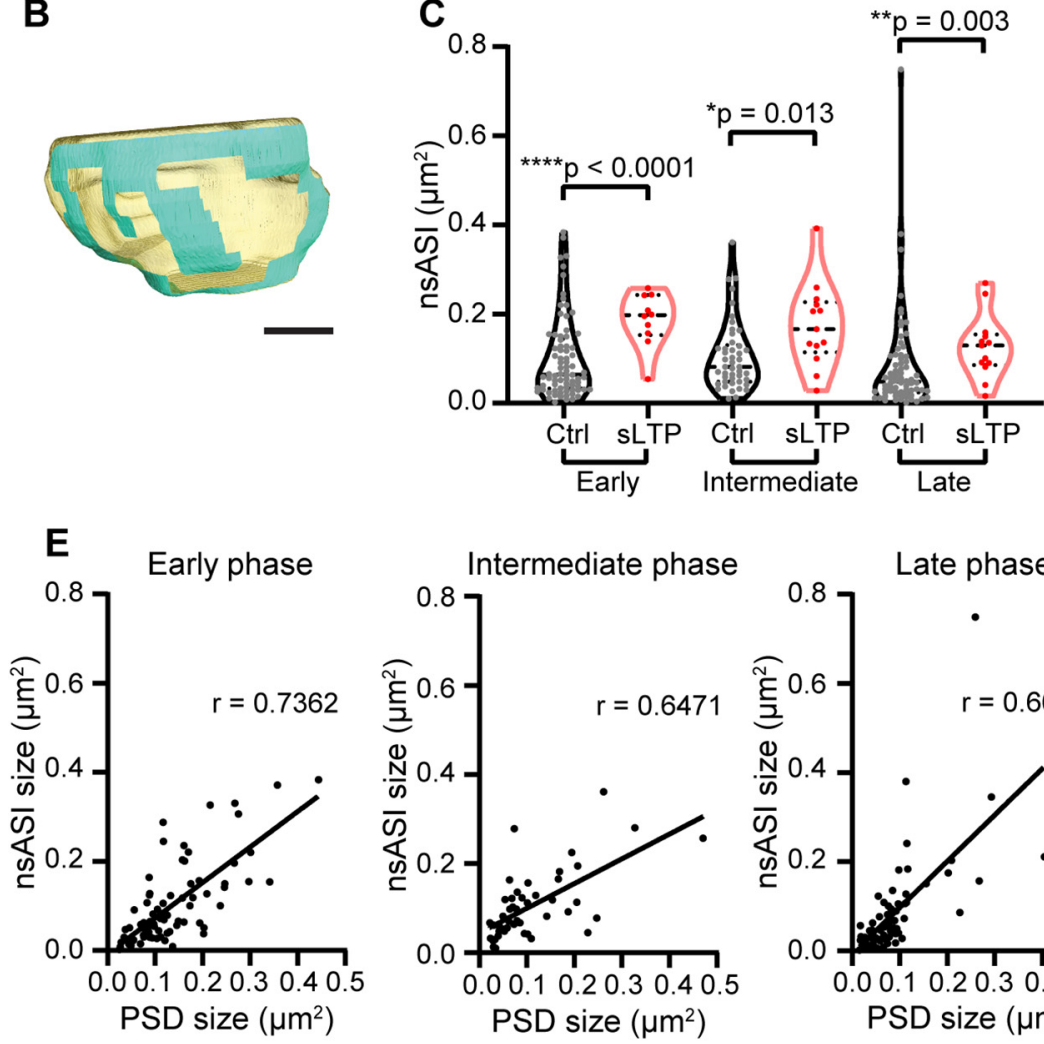

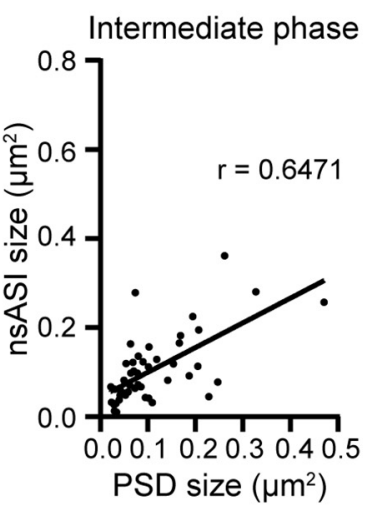

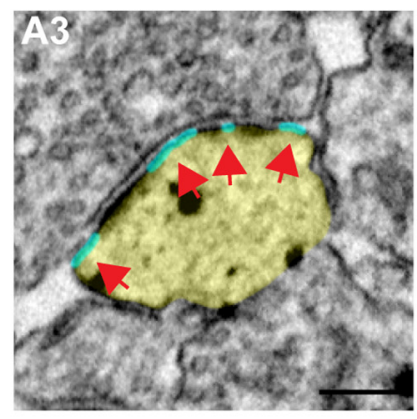
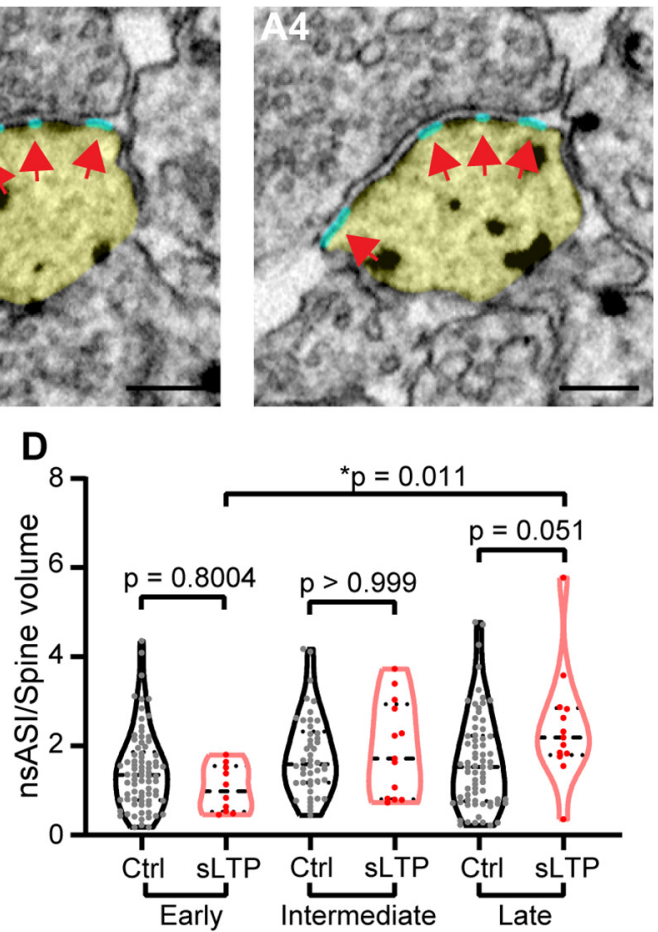

$\mathbf{F}$

Figure 6. SLTP induces sustained expansion of nSASI. $\boldsymbol{A}, \boldsymbol{B}$, Serial images of an example spine $(\boldsymbol{A})$ and $3 D$ reconstruction of the example spine $(\boldsymbol{B})$ with spine head shown in yellow and nsASI area shown in cyan, pointed out by red arrows. Scale bar, $200 \mathrm{~nm}$. C, nsASI size at early, intermediate, and late phases of sLTP. Welch's $t$ test on log-transformed data. D, Ratio of nsASI area to spine volume at early, intermediate, and late phases of sLTP for sLTP and control (Ctrl) spines. Two-way ANOVA followed by Bonferroni's post hoc test was used for comparison between control and SLTP spines, and Tukey's post hoc test was used for comparison among different phases. E, nsASI size is positively correlated with PSD size in control spines at all phases of sLTP. $r$, Pearson's correlation coefficient. $\boldsymbol{F}$, Ratio of nsASI area to PSD area at early, intermediate, and late phases of sLTP. Welch's $t$ test on log-transformed data. ${ }^{*} p<0.05$, ${ }^{* *} p<0.01$, ${ }^{* * *} p<$ $0.001,{ }^{* * *} p<0.0001$.

ASI consists of synaptic area, which contains PSD, and nonsynaptic area (nsASI), which lacks PSD. We found that nsASI expanded immediately after sLTP induction and maintained at a larger size until the late phase (Fig. $6 A-C$, Table 1). Since nsASI may also be able to support synaptic transmission (Ventura and Harris, 1999), this expanded nsASI may contribute to synaptic strengthening during LTP.

Similar to analyses of PSD and ASI area, we compared the growth of nsASI and spine volume by calculating the ratio of nsASI area to spine volume at different time points (Fig. 6D). Two-away ANOVA for stimulation conditions (control and sLTP) and time points (early, intermediate, and late) showed that the datasets were significantly different over time points ( $p=0.0048)$. Unlike PSD, post hoc multiple-comparison analyses showed that sLTP spines had trended to a larger ratio in the late phase $(p=0.0506)$, but not in the early phase $(p=0.8004)$ and intermediate phase $(p=1.0)$. The sLTP spines showed a significant increase in the ratio in the late phase, compared with the early phase ( $p=0.011$; Table 2). Thus, these results suggest that nsASI area increases immediately, similar to spine volume growth, and trends to exceed the spine volume growth at the late phase. Furthermore, the size of the nsASI area was proportional to that of the PSD area in control spines (Fig. 6E), which is consistent with a previous report (Ventura and Harris, 1999). However, the ratio of nsASI area to PSD area was higher in SLTP spines than in control spines in all sLTP phases, further proving an uneven expansion of synaptic and nonsynaptic spine membrane after sLTP induction (Fig. 6F).

\section{Discussion}

In this study, we developed an efficient method for analyzing ultrastructural changes of single dendritic spines after input-specific sLTP. Using this method, we found that PSD complexity 
increased within 2-3 min by nanometer-scale structural changes, including perforation and segmentation. In particular, a large fraction of PSDs showed segmentation into two to three segments during the early, intermediate, and late phases of sLTP. Associated with these changes, PSD size increased during the early phase of sLTP, although it was not as pronounced as changes in spine volume changes. On the other hand, our results suggested that the nsASI membrane underwent rapid, pronounced, and sustained expansion, which was proportional to the spine volume growth in the early and intermediate phases but exceeded the spine volume growth at the late phase. Our data indicated that the total increase in ASI area (= nsASI + PSD areas) during sLTP was majorly contributed by the nsASI area expansion.

Previously ultrastructural analyses following sLTP induced by uncaging have been performed using serial sectioned TEM (Bosch et al., 2014; Meyer et al., 2014). However, perhaps because of low yield, these studies restricted their analyses on PSD area size and spine volume. Our methods using ATUMtome serial sectioning and SEM imaging allowed us to achieve correlation more efficiently and to perform detailed analyses of the nanostructure of PSDs and spines. Although SEM imaging generally has a slight disadvantage in terms of resolution, our usage of the advanced detector system visualized sufficient ultrastructural details for the current study.

Organotypic slices have been used in numerous studies to study structural synaptic plasticity and functional synaptic plasticity (Maletic-Savatic et al., 1999; Matsuzaki et al., 2004; Kopec et al., 2007; De Roo et al., 2008; Lee et al., 2009; Makino and Malinow, 2009; Bosch et al., 2014; Meyer et al., 2014). Though the ultrastructure might be different in organotypic slices from those in acute slices and living animals, we chose to use organotypic slices because of their easy operation and stability to produce good ultrastructure preservation. Spine volume in control spines in the late phase was significantly smaller than that in the early phase (ANOVA on log-transformed data, $p=0.002$ ). This is likely because of the incubation of slices in ACSF containing MNI-glutamate and TTX for a long time. This trend was also observed in spines on different neurons located away from the stimulated spines $(>10 \mu \mathrm{m}$; Fig. $1 H)$. Previous studies also observed changes in ultrastructure after long-term incubation of slices with ACSF, including reduction in PSD area (Bell et al., 2014; Meyer et al., 2014) and active zone size (Bell et al., 2014).

It is known that LTP induction, either by glutamate uncaging or electrical stimulation, is associated with a rapid expansion of spine head volume coupled with an increase in postsynaptic sensitivity (Lang et al., 2004; Matsuzaki et al., 2004; Harvey et al., 2008; Yang et al., 2008b; Lee et al., 2009; Bosch et al., 2014) as well as presynaptic bouton volume change (Meyer et al., 2014) and presynaptic structure reorganization (Bell et al., 2014). The change of spine head volume is similar to the onset and time course of electrophysiological LTP indicated by AMPAR-mediated current (Matsuzaki et al., 2004; Kopec et al., 2006). However, it has been reported that the potentiation of PSD size is much slower than that of volume change, apparently inconsistent with rapid potentiation of AMPAR-mediated current (Bosch et al., 2014).

Our finding of the rapid complication of PSD nanostructure in the early phase, as well as in the intermediate and late phases, associated with a rapid expansion of nsASI area in these phases may be a mechanism underlying rapid recruitment of AMPARs to the synapse. AMPARs exist in extrasynaptic membrane near the PSD (Tao-Cheng et al., 2011; Nair et al., 2013) and likely were able to support synaptic transmission (Franks et al., 2003; Tardin et al., 2003), although their contribution to synaptic transmission is expected to be smaller (Franks et al., 2003). In particular, extrasynaptic AMPARs may play even more significant roles in LTP since LTP is known to be associated with a greater amount of glutamate release (Dolphin et al., 1982; Bekkers and Stevens, 1990; Malinow and Tsien, 1990; Zakharenko et al., 2001). This idea is consistent with previous electrophysiological experiments showing rapid recruitment of AMPARs to perisynaptic sites before the full expression of LTP (Yang et al., 2008a).

We induced sLTP at single spines with glutamate uncaging by delivering $2 \mathrm{P}$ laser pulses located $\sim 0.5 \mu \mathrm{m}$ from the tip of the spine head in GFP-expressing CA1 pyramidal neurons in organotypic hippocampal slices (Fig. $1 A-C$, Extended Data Fig. 1-1A; Matsuzaki et al., 2001, 2004; Harvey et al., 2008). This protocol is known to increase AMPAR-evoked current on the spine (Matsuzaki et al., 2004; Harvey et al., 2008). While this protocol is likely to activate extrasynaptic receptors (Franks et al., 2003), the plasticity depends on NMDAR and CaMKII, but not on mGluR, in a manner similar to that of electrophysiological LTP (Matsuzaki et al., 2004; Lee et al., 2009; Chang et al., 2017; Murakoshi et al., 2017). However, since previous studies suggest that metabotropic receptors (mGluRs) exist in perisynaptic areas within $\sim 60 \mathrm{~nm}$ of synapses (Luján et al., 1996), the expansion of nsASI area observed in all phases of LTP in our study may cause enhanced mGluR function after LTP induction.

We observed that two sLTP spines containing segmented PSDs are innervated by multiple presynaptic boutons. An increase of multi-innervated spines has been reported to be associated with LTP (Nikonenko et al., 2003; Radwanska et al., 2011; Giese et al., 2015). However, probably because of the rare existence of multi-innervated spines ( $\sim 1 \%$ of total spines), it has rarely been reported in previous population EM studies that analyzed the ultrastructural correlates for LTP. Since LTP is known to be correlated with a larger number of synapses in CA1 neurons (Tominaga-Yoshino et al., 2008; Watson et al., 2016), the rapid multi-innervated spines formation may serve as the basis for new synapse formation during SLTP. The multi-innervated spines observed in our experiments that synapsed with different presynaptic boutons likely support the theory of new bouton recruitment instead of spine splitting during LTP (Fiala et al., 2002).

One potential caveat of this study is that the uncaging laser irradiation may have caused some changes in the ultrastructure of dendritic spines. While previous studies have suggested that there is minimum impact on the electrophysiological properties from an uncaging laser (Matsuzaki et al., 2001, 2004; Lee et al., 2009; Murakoshi et al., 2011), this point remains to be resolved in future work.

Overall, our ultrastructural study revealed a time course of coordinated synaptic and extrasynaptic ultrastructural changes during sLTP induced at a single spine. Our results suggest that the combination of rapid and sustained increases in PSD complexity and nsASI membrane, as well as slow development of PSD size, are all likely substrates of synaptic potentiation at different temporal phases.

\section{References}

Bekkers JM, Stevens CF (1990) Presynaptic mechanism for long-term potentiation in the hippocampus. Nature 346:724-729.

Bell ME, Bourne JN, Chirillo MA, Mendenhall JM, Kuwajima M, Harris KM (2014) Dynamics of nascent and active zone ultrastructure as synapses 
enlarge during long-term potentiation in mature hippocampus. J Comp Neurol 522:3861-3884.

Bosch M, Castro J, Saneyoshi T, Matsuno H, Sur M, Hayashi Y (2014) Structural and molecular remodeling of dendritic spine substructures during long-term potentiation. Neuron 82:444-459.

Chang J-Y, Parra-Bueno P, Laviv T, Szatmari EM, Lee S-JR, Yasuda R (2017) CaMKII autophosphorylation is necessary for optimal integration of $\mathrm{Ca} 2+$ signals during LTP induction, but not maintenance. Neuron 94:800-808.e4.

De Roo M, Klauser P, Muller D (2008) LTP promotes a selective long-term stabilization and clustering of dendritic spines. PLoS Biol 6:e219.

Desmond NL, Levy WB (1986) Changes in the numerical density of synaptic contacts with long-term potentiation in the hippocampal dentate gyrus. J Comp Neurol 253:466-475.

Dolphin AC, Errington ML, Bliss TVP (1982) Long-term potentiation of the perforant path in vivo is associated with increased glutamate release. Nature 297:496-497.

Fiala JC, Allwardt B, Harris KM (2002) Dendritic spines do not split during hippocampal LTP or maturation. Nat Neurosci 5:297-298.

Franks KM, Stevens CF, Sejnowski TJ (2003) Independent sources of quantal variability at single glutamatergic synapses. J Neurosci 23:3186-3195.

Giese KP, Aziz W, Kraev I, Stewart MG (2015) Generation of multi-innervated dendritic spines as a novel mechanism of long-term memory formation. Neurobiol Learn Mem 124:48-51.

Gogolla N, Galimberti I, DePaola V, Caroni P (2006) Preparation of organotypic hippocampal slice cultures for long-term live imaging. Nat Protoc 1:1165-1171.

Harris KM, Stevens JK (1989) Dendritic spines of CA 1 pyramidal cells in the rat hippocampus: serial electron microscopy with reference to their biophysical characteristics. J Neurosci 9:2982-2997.

Harris KM, Jensen FE, Tsao B (1992) Three-dimensional structure of dendritic spines and synapses in rat hippocampus (CA1) at postnatal day 15 and adult ages: implications for the maturation of synaptic physiology and long-term potentiation. J Neurosci 12:2685-2705.

Harvey CD, Svoboda K (2007) Locally dynamic synaptic learning rules in pyramidal neuron dendrites. Nature 450:1195-1200.

Harvey CD, Yasuda R, Zhong H, Svoboda K (2008) The spread of Ras activity triggered by activation of a single dendritic spine. Science 321:136-140.

Hayashi-Takagi A, Yagishita S, Nakamura M, Shirai F, Wu YI, Loshbaugh AL, Kuhlman B, Hahn KM, Kasai H (2015) Labelling and optical erasure of synaptic memory traces in the motor cortex. Nature 525:333-338.

Hedrick NG, Harward SC, Hall CE, Murakoshi H, McNamara JO, Yasuda R (2016) Rho GTPase complementation underlies BDNF-dependent homo- and heterosynaptic plasticity. Nature 538:104-108.

Hruska M, Henderson N, Le Marchand SJ, Jafri H, Dalva MB (2018) Synaptic nanomodules underlie the organization and plasticity of spine synapses. Nat Neurosci 21:671-682.

Kamasawa N, Sun Y, Mikuni T, Guerrero-Given D, Yasuda R (2015) Correlative ultrastructural analysis of functionally modulated synapses using automatic tape-collecting ultramicrotome - SEM array tomography. Microsc Microanal 21:1271-1272.

Kopec CD, Li B, Wei W, Boehm J, Malinow R (2006) Glutamate receptor exocytosis and spine enlargement during chemically induced long-term potentiation. J Neurosci 26:2000-2009.

Kopec CD, Real E, Kessels HW, Malinow R (2007) GluR1 links structural and functional plasticity at excitatory synapses. J Neurosci 27:1370613718.

Lang C, Barco A, Zablow L, Kandel ER, Siegelbaum S. a, Zakharenko SS (2004) Transient expansion of synaptically connected dendritic spines upon induction of hippocampal long-term potentiation. Proc Natl Acad Sci U S A 101:16665-16670.

Lee S-JR, Escobedo-Lozoya Y, Szatmari EM, Yasuda R (2009) Activation of CaMKII in single dendritic spines during long-term potentiation. Nature 458:299-304.

Loewenstein Y, Kuras A, Rumpel S (2011) Multiplicative dynamics underlie the emergence of the log-normal distribution of spine sizes in the neocortex in vivo. J Neurosci 31:9481-9488.

Luján R, Nusser Z, Roberts JDB, Shigemoto R, Somogyi P (1996) Perisynaptic location of metabotropic glutamate receptors mGluR1 and mGluR5 on dendrites and dendritic spines in the rat hippocampus. Eur J Neurosci 8:1488-1500.
MacGillavry HD, Song Y, Raghavachari S, Blanpied TA (2013) Nanoscale scaffolding domains within the postsynaptic density concentrate synaptic ampa receptors. Neuron 78:615-622.

Makino H, Malinow R (2009) AMPA receptor incorporation into synapses during LTP: the role of lateral movement and exocytosis. Neuron 64:381-390.

Maletic-Savatic M, Malinow R, Svoboda K (1999) Rapid dendritic morphogenesis in CA1 hippocampal dendrites induced by synaptic activity. Science 283:1923-1927.

Malinow R, Tsien RW (1990) Presynaptic enhancement shown by whole-cell recordings of long-term potentiation in hippocampal slices. Nature 346:177-180.

Masch J-M, Steffens H, Fischer J, Engelhardt J, Hubrich J, KellerFindeisen J, D'Este E, Urban NT, Grant SGN, Sahl SJ, Kamin D, Hell SW (2018) Robust nanoscopy of a synaptic protein in living mice by organic-fluorophore labeling. Proc Natl Acad Sci U S A 115:E8047-E8056.

Matsuzaki M, Ellis-Davies GC, Nemoto T, Miyashita Y, Iino M, Kasai H (2001) Dendritic spine geometry is critical for AMPA receptor expression in hippocampal CA1 pyramidal neurons. Nat Neurosci 4:1086-1092.

Matsuzaki M, Honkura N, Ellis-Davies GCR, Kasai H (2004) Structural basis of long-term potentiation in single dendritic spines. Nature 429:761-766

Meyer D, Bonhoeffer T, Scheuss V (2014) Balance and stability of synaptic structures during synaptic plasticity. Neuron 82:430-443.

Murakoshi H, Wang H, Yasuda R (2011) Local, persistent activation of Rho GTPases during plasticity of single dendritic spines. Nature 472:100-104

Murakoshi H, Shin ME, Parra-Bueno P, Szatmari EM, Shibata ACE, Yasuda R (2017) Kinetics of endogenous CaMKII required for synaptic plasticity revealed by optogenetic kinase inhibitor. Neuron 94:37-47.e5.

Nair D, Hosy E, Petersen JD, Constals A, Giannone G, Choquet D, Sibarita JB (2013) Super-resolution imaging reveals that AMPA receptors inside synapses are dynamically organized in nanodomains regulated by PSD95. J Neurosci 33:13204-13224.

Nikonenko I, Jourdain P, Muller D (2003) Presynaptic remodeling contributes to activity-dependent synaptogenesis. J Neurosci 23:8498-8505.

Oh WC, Parajuli LK, Zito K (2015) Heterosynaptic structural plasticity on local dendritic segments of hippocampal CA1 neurons. Cell Rep 10:162169.

Ostroff LE, Fiala JC, Allwardt B, Harris KM (2002) Polyribosomes redistribute from dendritic shafts into spines with enlarged synapses during LTP in developing rat hippocampal slices. Neuron 35:535-545.

Radwanska K, Medvedev NI, Pereira GS, Engmann O, Thiede N, Moraes MFD, Villers A, Irvine EE, Maunganidze NS, Pyza EM, Ris L, Szymańska M, Lipiński M, Kaczmarek L, Stewart MG, Giese KP (2011) Mechanism for long-term memory formation when synaptic strengthening is impaired. Proc Natl Acad Sci U S A 108:1847118475.

Sala C, Segal M (2014) Dendritic spines: the locus of structural and functional plasticity. Physiol Rev 94:141-188.

Sun Y, Thomas C, Mikuni T, Guerrero-Given D, Yasuda R, Kamasawa N (2020) Correlative ultrastructural analysis of functionally modulated using automated tape-collecting ultramicrotome and SEM array tomography. Vol Microsc 155:121-149.

Tao-Cheng J-H, Crocker VT, Winters CA, Azzam R, Chludzinski J, Reese TS (2011) Trafficking of AMPA receptors at plasma membranes of hippocampal neurons. J Neurosci 31:4834-4843.

Tardin C, Cognet L, Bats C, Lounis B, Choquet D (2003) Direct imaging of lateral movements of AMPA receptors inside synapses. EMBO J 22:4656-4665.

Tominaga-Yoshino K, Urakubo T, Okada M, Matsuda H, Ogura A (2008) Repetitive induction of late-phase LTP produces long-lasting synaptic enhancement accompanied by synaptogenesis in cultured hippocampal slices. Hippocampus 18:281-293.

Toni N, Buchs PA, Nikonenko I, Bron CR, Muller D (1999) LTP promotes formation of multiple spine synapses between a single axon terminal and a dendrite. Nature 402:421-425. 
Toni N, Buchs PA, Nikonenko I, Povilaitite P, Parisi L, Muller D (2001) Remodeling of synaptic membranes after induction of long-term potentiation. J Neurosci 21:6245-6251.

Van Harreveld A, Fifkova E (1975) Swelling of dendritic spines in the fascia dentata after stimulation of the perforant fibers as a mechanism of posttetanic potentiation. Exp Neurol 49:736-749.

Ventura R, Harris KM (1999) Three-dimensional relationships between hippocampal synapses and astrocytes. J Neurosci 19:6897-6906.

Watson DJ, Ostroff L, Cao G, Parker PH, Smith H, Harris KM (2016) LTP enhances synaptogenesis in the developing hippocampus. Hippocampus 26:560-576.

Wegner W, Mott AC, Grant SGN, Steffens H, Willig KI (2018) In vivo STED microscopy visualizes PSD95 sub-structures and morpho- logical changes over several hours in the mouse visual cortex. Sci Rep 8:219.

Woods G, Zito K (2008) Preparation of gene gun bullets and biolistic transfection of neurons in slice culture. J Vis $\operatorname{Exp}(12): \mathrm{e} 675$

Yang Y, Wang X-BB, Frerking M, Zhou Q (2008a) Delivery of AMPA receptors to perisynaptic sites precedes the full expression of long-term potentiation. Proc Natl Acad Sci U S A 105:11388-11393.

Yang Y, Wang X, Frerking M, Zhou Q (2008b) Spine expansion and stabilization associated with long-term potentiation. J Neurosci 28:5740-5751.

Zakharenko SS, Zablow L, Siegelbaum SA (2001) Visualization of changes in presynaptic function during long-term synaptic plasticity. Nat Neurosci 4:711-717. 